\title{
Changes in Midbrain Pain Receptor Expression, Gait and Behavioral Sensitivity in a Rat Model of Radiculopathy
}

\author{
Priscilla Y. Hwang ${ }^{1}$, Kyle D. Allen ${ }^{1,2}$,Mohammed F. Shamji ${ }^{1,3}$, Liufang Jing ${ }^{1}$, Brian A. Mata ${ }^{2}$, \\ Mostafa A. Gabr ${ }^{2}$, Janet L. Huebner ${ }^{4}$, Virginia B. Kraus ${ }^{4}$, William J. Richardson ${ }^{2}$ and Lori A. Setton ${ }^{*}, 1,2$ \\ ${ }^{I}$ Department of Biomedical Engineering, Duke University, Durham, NC, USA \\ ${ }^{2}$ Department of Orthopaedic Surgery, Duke University Medical Center, Durham, NC, USA \\ ${ }^{3}$ Division of Neurosurgery, The Ottawa Hospital, Ottawa, ON, Canada \\ ${ }^{4}$ Division of Rheumatology and Immunology, Department of Medicine, Duke University Medical Center, Durham, NC, \\ USA
}

\begin{abstract}
Intervertebral disc herniation may contribute to inflammatory processes that associate with radicular pain and motor deficits. Molecular changes at the affected dorsal root ganglion (DRG), spinal cord, and even midbrain, have been documented in rat models of radiculopathy or nerve injury. The objective of this study was to evaluate gait and the expression of key pain receptors in the midbrain in a rodent model of radiculopathy. Radiculopathy was induced by harvesting tail nucleus pulposus (NP) and placing upon the right L5 DRG in rats (NP-treated, n=12). Tail NP was discarded in sham-operated animals $(\mathrm{n}=12)$. Mechanical allodynia, weight-bearing, and gait were evaluated in all animals over time. At 1 and 4 weeks after surgery, astrocyte and microglial activation was tested in DRG sections. Midbrain sections were similarly evaluated for immunoreactivity to serotonin $\left(5 \mathrm{HT}_{2 \mathrm{~B}}\right)$, mu-opioid ( $\mu$-OR), and metabotropic glutamate (mGluR4 and 5) receptor antibodies. NP-treated animals placed less weight on the affected limb 1 week after surgery and experienced mechanical hypersensitivity over the duration of the study. Astroctye activation was observed at DRGs only at 4 weeks after surgery. Findings for pain receptors in the midbrain of NP-treated rats included an increased expression of $5 \mathrm{HT}_{2 \mathrm{~B}}$ at 1 , but not 4 weeks; increased expression of $\mu$-OR and mGluR5 at 1 and 4 weeks (periaqueductal gray region only); and no changes in expression of mGluR4 at any point in this study. These observations provide support for the hypothesis that the midbrain responds to DRG injury with a transient change in receptors regulating pain responses.
\end{abstract}

Keywords: Dorsal root ganglion, gait, intervertebral disc, lumbar radiculopathy, metabotropic glutamate receptor, midbrain, mu-opioid receptor, serotonin receptor.

\section{INTRODUCTION}

Herniation of the intervertebral disc (IVD) is a common cause of low back pain and may be associated with nerve root dysfunction, known as radiculopathy. Features of radiculopathy can range from radiating neuropathic pain to paresthesias and sensory deficits to motor deficits, and the societal cost of this pathology involves over 660,000 hospital visits and over 325,000 surgeries for discectomy procedures annually in the USA [1]. The consequent mechanical compression and biochemical irritation of nearby neural structures seems to be mediated by heightened inflammatory and immune cytokine expression alongside inflammatory cell infiltration [2-7]. Indeed, upon systemic exposure, the nucleus pulposus (NP) component of IVD tissue initiates a specific immune response, likely a consequence of its immune privileged avascular location bounded by the anulus fibrosus (AF) [8, 9].

*Address correspondence to this author at the Department of Biomedical Engineering and Orthopaedic Surgery, Box 90281, Duke University, Durham, NC 27708, USA; Tel: (919) 660-5131; Fax: (919) 660-5362;

E-mail: setton@duke.edu
There is clear interest in identifying the cell populations affected by disc herniation-induced radiculopathy, and the role of neurotransmitters and their receptors that mediate the symptomatic and functional deficits of radiculopathy. Animal models based on compression injury of the dorsal root ganglion (DRG) demonstrate resultant allodynia and functional deficits in association with an elevated microglial activation in the spinal cord [10-14]. In addition, molecular and mRNA changes occur locally at the DRG or in the spinal cord implicating key inflammatory mediators (e.g., TNF- $\alpha$, IL-1, nitric oxide) and neuropeptides (e.g., substance P) following DRG compression injury or exposure to autologus NP [9, 15-23]. These observations suggest a hypothesis that neuroplastic remodeling of the spinal cord can contribute to or modulate the pain and symptoms of disc herniationassociated radiculopathy.

The goal of the current study was to expand the scope of the disc herniation associated radiculopathy model to understand not only the induced pain phenotype, but also the mechanisms underlying spontaneous functional recovery. In prior studies of a model of non-compressive disc herniation with autologous NP material, animals exhibited behavioral changes including heightened behavioral mechanical 
sensitivity, stance symmetry, and gait parameters including symmetry and force analysis. Divergent findings of gait symmetry andmechanical sensitivity suggested supraspinal modulation of the nociceptive phenotype and promoted investigation into mechanisms underlying that descending control. An important objective of the current study was to evaluate a role for modified neurotransmitter receptor expression in the central nervous system at the midbrain to test if neuroplastic remodeling can occur central subsequent to DRG injury or sensitization. Thus, transient changes were studied here in a rodent model of non-compressive disc herniation. Immunohistochemical markers of neuroinflammation at the DRG and the presence of a monocyte chemotactic protein (MCP-1) were quantified in the cerebrospinal fluid. The expression of serotonin, metabotropic glutamate, and mu-opioid receptors was characterized in the periaqueductal gray and red nucleus regions of the midbrain by immunohistochemistry.

\section{MATERIALS AND METHODOLOGY}

\section{Animal Model}

Male Sprague-Dawley rats $(\mathrm{n}=30,9$ months old) were divided into sham $(\mathrm{n}=12)$, NP-treated $(\mathrm{n}=12)$, and preoperative control $(\mathrm{n}=6)$ groups. The sham and NP-treated groups underwent a previously described operation [15] as approved by Duke University Institutional Animal Care and Use Committee (IACUC) while the pre-operative control animals served as non-surgical control tissue.

In brief, under pentobarbital anesthesia, rats underwent surgical exposure of proximal tail IVD in which a single disc was punctured, the NP was harvested via curette, and the tail was closed. In these same animals, a dorsal exposure of the right L5 DRG was accomplished by a partial unilateral laminotomy and medial facetectomy, with autologous NP either discarded (sham) or placed onto the exposed DRG (NP-treated). Two-layer closure was performed and animals were returned to solitary cage housing for 5-7 days, followed by group housing until sacrifice.

Animals in sham and NP-treated groups were studied longitudinally for gait, stance symmetry, and behavioral sensitivity at pre-operative and post-operative time points. Animals were then sacrificed at 7 or 28 days post surgery $(n=6$ per animal group for each time point) after which the cerebrospinal fluid, midbrain and DRG were harvested. The pre-operative control animals were procured and midbrain harvested only to serve as a control group.

\section{Geometric and Temporal Gait Descriptors}

To assess rodent gait, rat ambulation was recorded while freely exploring a custom-built gait arena (5'6" x 1'6") preoperatively and at 1 and 4 weeks after surgery, as previously described [15]. When a rat passed through the middle of the arena, a high-speed video camera was manually triggered to capture the rat's movement (Phantom V4.2, 200 frames per second; Vision Research, Wayne, NJ). Trials that contained a minimum of two complete gait cycles and a consistent velocity were saved for processing (5 trials per animal). Using custom code (DLTdataviewer), the video frame of foot-strike and toe-off events were determined viaby-hand digitization $[24,25]$. Using these data, percentage stance time imbalance and gait symmetry were calculated for each trial. Percentage stance time (also known as limb duty factor) is the percentage of time a limb is in stance for a given stride, or mathematically as stance time divided by stride time [26]. Gait symmetry is the offset between left and right foot-strikes in a limb pair for a given stride, or mathematically as the time between left and right foot-strikes divided by time between two left foot-strikes [26]. Rats commonly ambulate with balanced, symmetric gaits, represented mathematically by a difference between the left and right percentage stance times of 0 and a gait symmetry of approximately 0.5 ; a shift in these variables would indicate an unbalanced or asymmetric gait, respectively. Data for each group (sham, NP-treated) were compared to the mathematical definitions for balanced, symmetric gait using a repeated measures t-test on time with a Bonferroni correction; differences amongst treatment groups and between pre-operative and post-operative timepoints were analyzed using a two-way ANOVA with a post-hoc Tukey's test.

\section{Weight Distribution}

Hind limb weight distribution was determined preoperatively and at 1 and 4 weeks after surgery. An incapacitance meter (IITC, Inc.) was used to measure weight distribution on the left and right limb, and data was acquired during 5 second intervals (5 trials per rat). Weight distribution for each group was compared to the mathematical definitions for balanced weight distribution using a repeated measures t-test on time with a post-hoc Bonferroni correction (unbalanced distribution $\neq 50 \%$ weight on right limb); differences amongst groups (sham, NPtreated) and between pre-operative and post-operative timepoints were analyzed using a two-way ANOVA.

\section{Mechanical Sensitivity}

Mechanical paw withdrawal thresholds were determined preoperatively and at 1 and 4 weeks after surgery using an up-down protocol described by Chaplan and coworkers [27]. Briefly, rats were placed in a wire-bottom cage and von Frey filaments (Stoelting) were applied to the plantar surface of rat's hind paws. If paw withdrawal was observed, the next smallest filament was applied; if paw withdrawal was not observed, the next largest filament was applied. Using this up-down protocol, the $50 \%$ paw withdrawal threshold can be approximated; this threshold represents the mechanical force where paw withdrawal and stimulus tolerance are equally likely. A two-factor ANOVA followed by a post-hoc Tukey's test was used to compare differences amongst treatment groups (sham, NP treated) and between preoperative and post-operative timepoints for the affected limb.

\section{DRG Immunostaining}

At the time of dissection, lumbar DRGs from sham and NP-treated groups were dissected en bloc and placed in optical cutting temperature (OCT) embedding media (Sakura Finetek, Torrence CA) and frozen in liquid nitrogen until sectioning. Cryosections of DRG $(7 \mu \mathrm{m})$ were immunostained for glial fibrilary acidic protein (GFAP) to identify astrocytes. DRG sections were fixed in cold acetone $\left(10 \mathrm{~min},-20^{\circ} \mathrm{C}\right)$, permeabilized with $0.02 \%$ Triton- 100 , and incubated with blocking serum (30 min, 3.75\% BSA (Gibco) and $5 \%$ goat serum (Zymed, Invitrogen, Carlsbad, CA)) to 
minimize non-specific antibody binding. Sections were incubated with primary monoclonal mouse GFAP antibody (GFAP, SC-51908, Santa Cruz Biotechnology, Santa Cruz, $\mathrm{CA}$ ) diluted $50 \mathrm{x}$ in blocking serum (2 hours at room temperature), washed with PBS and incubated with Alexa Fluor 488-conjugated secondary antibodies (30 min at 200x dilution in blocking serum, Molecular Probes, Invitrogen).

Separate DRG sections were immunostained to detect the presence of ionized calcium binding adaptor molecule 1 (Iba1) to determine presence of macrophages. DRG cryosections were fixed in cold methanol and acetone mixture $(1: 1)$ for $5 \mathrm{~min}(-20 \circ \mathrm{C})$, permeabilized with $0.5 \%$ Triton-100 (15 min, room temperature) and incubated with blocking serum (30 min, 1\% BSA, 0.25\% Triton-100, \& 5\% goat serum). Sections were incubated with rabbit polyclonal Iba1 antibody (Wako, 019-19741) with a 250x dilution in blocking serum overnight. DRG sections were washed with PBS and incubated with Alexa Fluor 488-conjugated secondary antibodies $(30 \mathrm{~min}$ at $200 \mathrm{x}$ dilution in blocking serum, Molecular Probes).

All stained DRG sections were counterstained with 0.5 $\mathrm{mg} / \mathrm{ml}$ propidium iodide (Sigma-Aldrich) at room temperature for 15 minutes to stain the cell nuclei and mounted with GVA mounting medium (Zymed, Invitrogen).

Stained tissue sections were imaged using a confocal laser scanning microscope (Zeiss LSM 510, 20x objective; Zeiss, Jena, Germany) with 20x magnification. Multiple digital images (526x526) were acquired for each DRG sample and evaluated by two blinded graders coming to consensus for fluorescence staining uniformity and intensity with the following ordinal scale: 0 (no stain), 1 (moderate, non-uniform stain), 2 (moderate, uniform stain), 3 (intense stain) for each image.

Grades of immunohistochemical staining for GFAP and Iba1 were tested for evidence of differences between 1 and 4 weeks time period for sham and NP-treated animals using Kruskal-Wallis one-way analysis of variance with a 0.05 level of significance. When significance was observed, posthoc Wilcoxon Rank Sum Tests were conducted to detect differences in time for each group.

\section{Midbrain Immunostaining}

At the time of sacrifice, midbrains from all animals were dissected en bloc and placed in optical cutting temperature (OCT) embedding media (Sakura Finetek) and frozen in liquid nitrogen until sectioning. Cryosections of midbrain $(25 \mu \mathrm{m})$ were fixed in $4 \%$ formaldehyde $(10 \mathrm{~min}$, room temperature), permeabilized with $0.2 \%$ Triton $(10 \mathrm{~min})$, and incubated with blocking solution (30 min in $3.75 \%$ BSA and $5 \%$ goat serum, Invitrogen). Sections were incubated with monoclonal mouse serotonin receptor $2 \mathrm{~b}\left(5 \mathrm{HT}_{2 \mathrm{~b}}, 556334\right.$, BD Biosciences Pharmingen, San Jose, CA ) diluted 100x in blocking solution for 60 minutes. Samples were then washed with PBS and incubated with an AlexaFluor 488-conjugated secondary antibody (Molecular Probes) diluted 100x in blocking buffer for 30 minutes. Cell nuclei were counterstained using $0.5 \mathrm{mg} / \mathrm{ml}$ propidium iodide $(20 \mathrm{~min}$, Sigma-Aldrich) and slides were mounted with GVA mounting solution (Invitrogen).
A separate protocol was used to immunolabel midbrain sections with antibodies against metabotropic glutamate and $\mu$-opoid receptors as described here. Cryosections of midbrain $(25 \mu \mathrm{m})$ were fixed in $10 \%$ formaldehyde $(30 \mathrm{~min}$, room temperature), quenched in peroxo-block ( $2 \mathrm{~min}, 1$ part $30 \% \mathrm{H}_{2} \mathrm{O}_{2}: 9$ parts methanol), and incubated in ready-to-use $2.5 \%$ normal horse blocking serum (20 min, ImmPRESS Universal Reagent kit: MP7500, Vector labs, Burlingame, CA). Samples were then incubated for 60 minutes with the following primary antibodies in blocking serum: rabbit polyclonal metabotropic glutamate receptor 5 (diluted 200x, mGluR5; ab53090, Abcam Inc, Cambridge, MA), rabbit polyclonal metabotropic glutamate receptor 4 (diluted 400x, mGluR4; 06-765, Upstate, Lake Placid, NY), or rabbit polyclonal $\mu$-opioid receptor (diluted 100x, $\mu-\mathrm{OR}, 44308 \mathrm{G}$, Biosource, Camarillo CA). Samples were washed in PBS and incubated with ImmPRESS Peroxidase Universal anti mouse/rabbit Ig reagent $(30 \mathrm{~min}$, ImmPRESS Universal Reagent kit), washed again and incubated with diaminobenzideine-DAB solution (30 min, SK-4100, Vector labs). Cell nuclei were counterstained using Methyl Green $\left(\mathrm{H}-3402\right.$, Vector labs) for 5 minutes $\left(60^{\circ} \mathrm{C}\right)$ and mounted with GVA mounting solution (Invitrogen).

Immunostained sections of the rat midbrain were imaged with a confocal laser scanning microscope $\left(5 \mathrm{HT}_{2 \mathrm{~b}}\right.$ receptor only; Zeiss LSM 510, 20x objective; Zeiss, Jena, Germany) or a light microscope (Nikon Eclipse E600 POL, 4x objective; Nikon Instruments Inc, USA). Digital images were acquired of midbrain regions and analyzed as follows. For the $5 \mathrm{HT}_{2 \mathrm{~b}}$ receptor, images were thresholded on red and green channels to highlight labeled cell nuclei and associated staining. The numbers of cells and positively labeled cells were counted to determine the percent of positively stained cells.

To analyze intensity of positive staining for all other receptors, the periaqueductal gray (PAG) or red nucleus (RN) regions of the midbrain were first identified. Using Matlab, 526x526 pixel sized regions within the PAG or RN were obtained and converted to grayscale images. Images were then thresholded against a corresponding negative control to highlight only positively stained cells. After thresholding, images were converted to black and white images in order to calculate percent of positively stained pixels in Matlab.

Midbrain data (\% positively labeled cells or \% positively labeled pixels) for each pain receptor was analyzed for differences amongst pre-operative control, shamand NPtreated groups at day 0 , week 1 and week 4 time points by two-way analysis of variance (2-way ANOVA) at a 0.05 level of significance. Tukey's post-hoc analysis was performed when significance was observed.

\section{Cerebrospinal Fluid (CSF) Biomarker Assessment}

Prior studiesdemonstrated elevated expression of monocyte-chemoattractant protein (MCP-1) in multiple sclerosis [28], acute spinal cord injury [29], and traumatic brain injury [30]. To investigate changes in CSF concentrations of MCP-1, CSF was collected at the time of sacrifice through a 23 gauge needle puncture of the $4^{\text {th }}$ ventricle. Due to technical limitations, CSF samples were collected from 3 NP-treated rats at 1 week, 5 NP-treated rats 
at 4 weeks, 6 sham rats at 1 week, and 4 sham rats at 4 weeks. Levels of CSF MCP-1 were quantified using a commercially available ELISA (ERMCP-1C, Thermo Fisher Scientific, Waltham MA, USA) following the manufacturer's instructions. Briefly, MCP-1 reference and rat CSF samples, diluted 1:25, were incubated in microwells coated with MCP-1 binding protein. Data were analyzed via an unbalanced two-factor, full-factorial ANOVA.

\section{RESULTS}

\section{Mechanical Sensitivity}

Mechanical allodynia was observed in the affected limb of animals exposed to autologous NP (Table 1). Lower values for $50 \%$ paw withdrawal thresholds among NPtreated animals were observed when compared to preoperative testing, sham, and their own contralateral hindlimbs. This lower mechanical withdrawal threshold occurred early at 1 week and persisted through to the 4 week evaluation $(p<0.05$, Table 1). There was no evidence of a change in mechanical withdrawal thresholds in the affected limb of sham animals $(p>0.05)$, nor was there evidence of a change in mechanical withdrawal thresholds in the contralateral limb for NP-treated or sham animals (data not shown).

\section{Weight Distribution}

NP-treated animals had a weight-distribution imbalance at 1 week ( $p<0.05$, Table 1), which was significantly different from the weight distribution observed pre-surgery $(p<0.02$, Table 1). By week 4, balanced weight-distribution returned in NP-treated animals. Weight-distribution in sham animals remained balanced and did not vary from presurgery values.

\section{Temporal Gait Descriptors}

NP-treated animals used imbalanced, asymmetric gaits at 1 week (Table 1). Gait asymmetry and imbalance of limb duty factor was observed on the affected limb. Percentage stance times were significantly shorter on the affected limb relative to the contralateral limb (percentage stance time imbalance $>0 \%, p<0.05)$, and gait symmetry indicated that left and right foot-strikes were not equally spaced in time (gait symmetry $\neq 50 \%, p<0.05$ ). This temporal gait profile was significantly different from both pre-surgery values and sham controls at 1 week. By 4 weeks, NP-treated animals returned to balanced, symmetric gaits. Gait asymmetry and imbalance were not observed for sham animals.

\section{DRG Immunostaining}

GFAP expression in the DRG was found to be higherin the DRGs of NP-treatedanimals at 4 weeks post-operatively as compared to the 1 -week timepoint $(p<0.0004)$ (Fig. 1), with no evidence of differences in GFAP staining for sham animals at either timepoint. Overall, the intensity of staining was found to be very low with mean values that correspond to moderate and non-uniform staining in the sham animals.

Very low levels of staining were observed in all DRGs for Ibal expression, with no evidence of differences between 1 and 4 week timepoints in either sham or NP-treated animals (Fig. 1).

\section{Midbrain Immunostaining}

Expression of serotonin $2 \mathrm{~b}$ receptor $\left(5 \mathrm{HT}_{2 \mathrm{~b}}\right)$ was increased at 1 week in NP-treated animals $(\mathrm{p}<0.0015 \mathrm{vs}$ unoperated controls) and returned to pre-operative control levels by 4 weeks (Fig. 2). Expression level of $5 \mathrm{HT}_{2 \mathrm{~b}}$ for sham animals remained the same level as pre-operative controls. Metabotropic glutamate receptor 5 expression (mGluR5) was increased in the PAG region of both sham $(p<0.0001 v s$ pre-operative controls) and NP-treated animals $(p<0.0001$ vs pre-operative controls) at one week (Fig. 3). The increase in expression level of mGluR5 in the PAG at one week for NP-treated animals was significantly higher than sham animals $(p<0.0001)$. By four weeks, mGluR5 expression returned to the same expression level as preoperative controls in sham animals, while expression levels in NP-treated animals remained elevated $(p<0.0001 v s$ preoperative controls and sham animals at 4 weeks). mGluR5 expression levels in the RN of sham and NP-treated animals was not significantly different from each other and remained the same level as pre-operative controls (Fig. 3). mGluR4 expression level of sham and NP-treated animals also remained the same as pre-operative controls at all time points (Fig. 4). Increased expression of $\mu$-opioid receptor ( $\mu$-OR) was observed after 1 and 4 weeks in sham animals $(p<0.0001 v s$ pre-operative controls) and NP-treated animals $(\mathrm{p}<0.0001$ vs pre-operative controls) as compared to preopreative control basal levels (Fig. 5). In NP-treated animals, expression levels of $\mu$-opioid receptor increased even above sham animals levels at the 4 week timepoint $(p<0.0001)$, but in $\mathrm{PAG}$ regions only. In the $\mathrm{RN}$ region, $\mu$-opioid receptor

Table 1. Data for Gait Parameters. All Data Shown are Mean \pm Standard Error

\begin{tabular}{|l|c|c|c|c|c|}
\hline & Pre-Operative & Sham (1 Week) & Sham (4 Weeks) & NP-Treated (1 Week) & NP-Treated (4 Weeks) \\
\hline \hline $\begin{array}{l}\text { Mechanical withdrawal threshold } \\
\text { (grams-force) }\end{array}$ & $14.6 \pm 1.2$ & $7.4 \pm 1.1$ & $11.8 \pm 2.1$ & $3.4 \pm 0.7^{* \perp}$ & $4.3 \pm 1.0^{*}$ \\
\hline $\begin{array}{l}\text { Weight-bearing } \\
\text { (\% weight on affected limb) }\end{array}$ & $49.5 \pm 1.0$ & $47.3 \pm 3.0$ & $50.0 \pm 0.7$ & $41.9 \pm 3.1^{* \forall}$ & $49.2 \pm 0.9$ \\
\hline $\begin{array}{l}\text { Percentage stance time imbalance } \\
\text { (\% stance time, left-right limb) }\end{array}$ & $0.2 \pm 0.4$ & $-0.6 \pm 0.9$ & $0.0 \pm 0.6$ & $4.1 \pm 1.4^{* \forall}$ & $1.5 \pm 1.7$ \\
\hline Gait symmetry & $49.3 \pm 0.4$ & $50.9 \pm 0.8$ & $50.7 \pm 0.5$ & $54.5 \pm 1.5^{* \forall}$ & $52.0 \pm 2.3$ \\
\hline
\end{tabular}

$\forall$ Imbalanced/asymmetric $(\mathrm{p}<0.05)$.

$*$ Different from pre-operative data $(\mathrm{p}<0.05)$.

\#Different from 1 week sham $(\mathrm{p}<0.05)$.

$\perp$ Different from 4 week sham $(\mathrm{p}<0.05)$ 

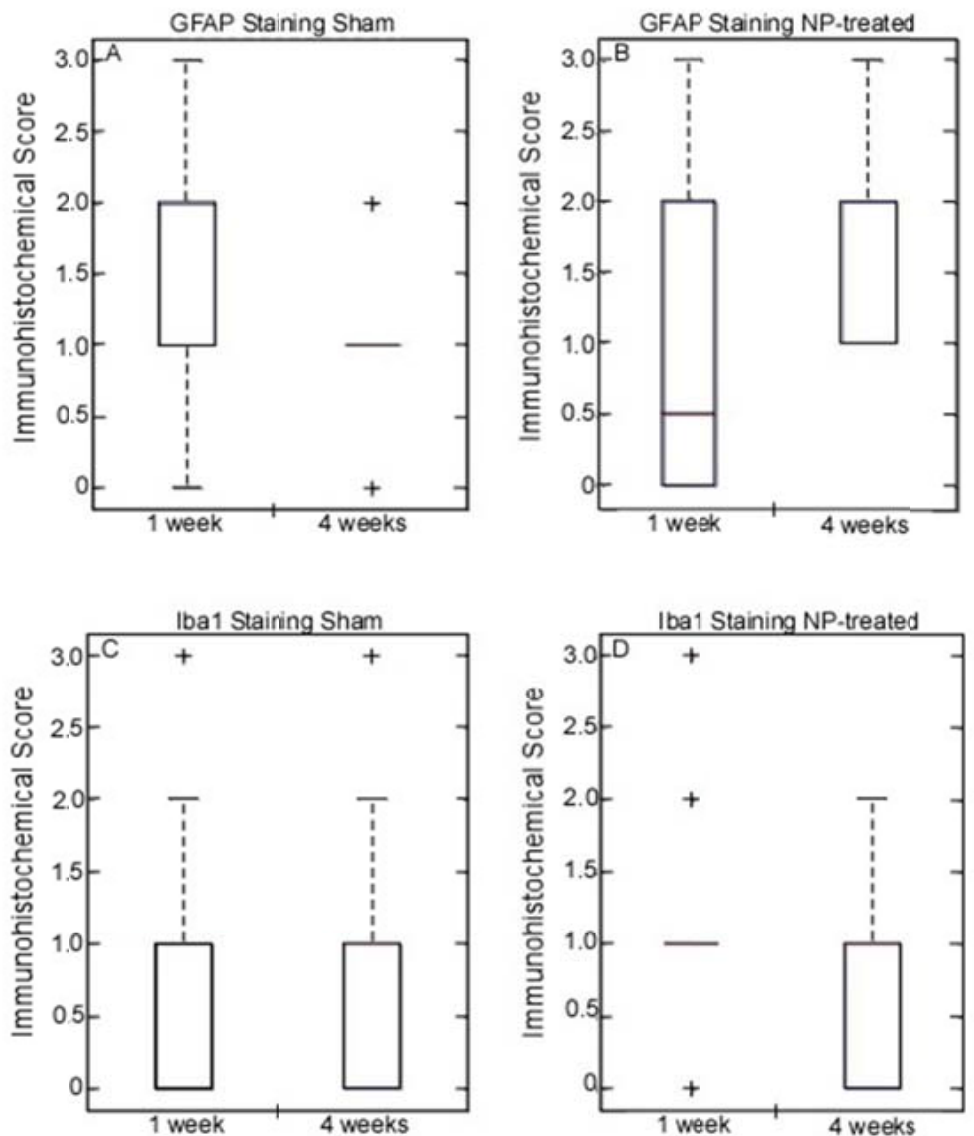

Fig. (1). DRG Immunostaining: Glial fibrillary acidic protein (GFAP) in DRG (top; graphs A-B): Increased expression was observed in NPtreated $\left({ }^{*} p<0.0004\right)$ animals at 4 weeks after surgery. Expression levels of GFAP in sham animals remained constant at all time points. Ionized calcium binding adaptor molecular 1 (Iba1) in DRG (bottom; graphs C-D): Expression level of Iba1 did not vary in sham or NPtreated animals at any time point.
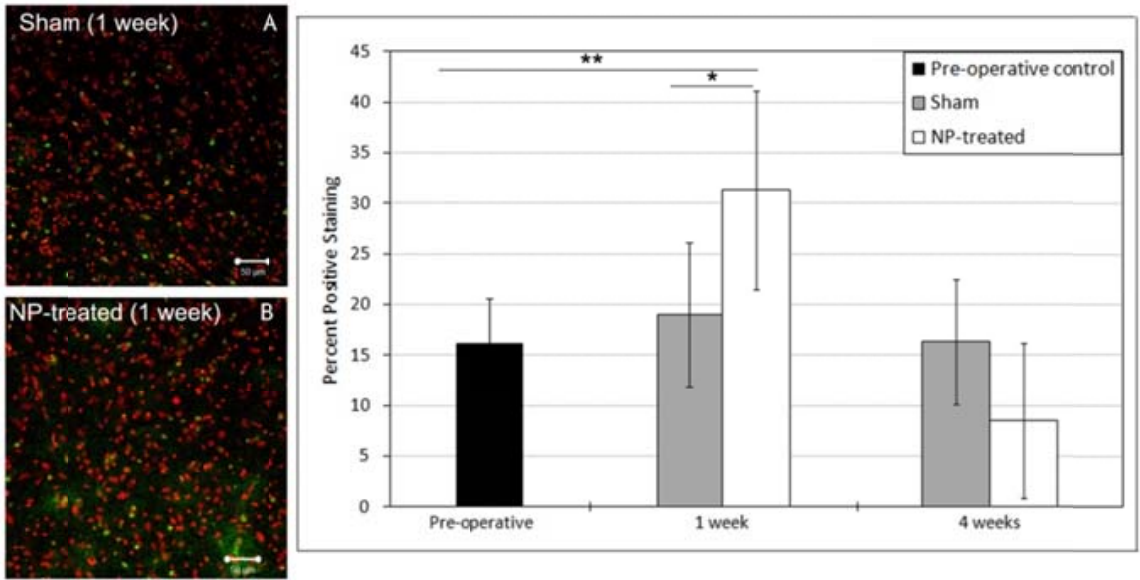

Fig. (2). Serotonin $2 \mathrm{~b}\left(5 \mathrm{HT}_{2 \mathrm{~b}}\right)$ Receptor Immunostaining: (left) Representative images of $5 \mathrm{HT}_{2 \mathrm{~b}}$ immunohistochemical images for sham (A) and NP-treated (B) 1 week after surgery (red=nuclei; green=positive staining; scale bar $=100 \mu \mathrm{m}$ ). Increased expression was observed in NPtreated $(* * p<0.015)$ animals at 1 week after surgery, but returned to pre-operative levels by 4 weeks post surgery. Expression level at 1 week for NP-treated animals was significantly different from sham animals $\left({ }^{*} p<0.015\right)$. Data are presented as mean \pm standard deviation.

expression levels did not change for sham or NP-treated animals (Fig. 5).

\section{Cerebrospinal Fluid (CSF) Biomarker}

MCP-1 was detectable in CSF of all rats in sham and NPtreated groups at $\mathrm{ng} / \mathrm{ml}$ levels (Fig. 6). When comparing
MCP-1 levels between sham and NP-treated groups, MCP-1 levels tended to be higher in NP treated rats as compared to sham-operated controls at the 4 week timepoint. The magnitude of difference in MCP-1 values was large between these two groups at 4 weeks post-operatively, nearly 2 -fold, although a statistically significant difference was not 

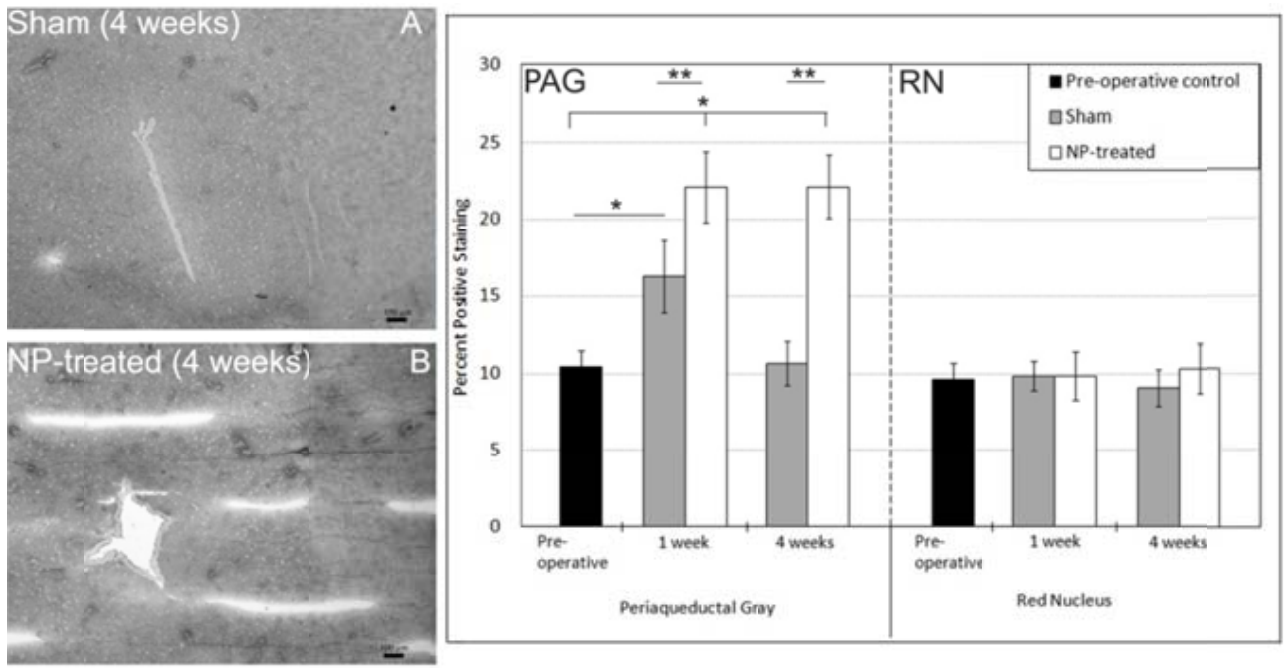

Fig. (3). Metabotropic Glutamate Receptor 5 (mGluR5) Immunostaining: PAG: (left) Representative images of mGluR5 immunohistochemical images for sham (A) and NP-treated (B) 4 weeks after surgery (dark spots represent positive staining; scale bar $=100$ $\mu \mathrm{m})$. Increased expression was observed in both sham $\left({ }^{*} p<0.0001\right)$ and NP-treated $\left({ }^{*} p<0.0001\right)$ animals at 1 week after surgery compared to unoperated controls. At one week post surgery, mGluR5 expression levels was significantly different in NP-treated compared to sham $(* * p<0.0001)$. Expression levels of mGluR5 in sham animals returned to expression levels of pre-operative controls at 4 weeks while NPtreated $(* * p<0.0001)$ animals continued to have increased expression of mGluR5. RN: Expression level of mGluR5 in the red nucleus did not vary in sham or NP-treated animals. Data are presented as mean \pm standard deviation.
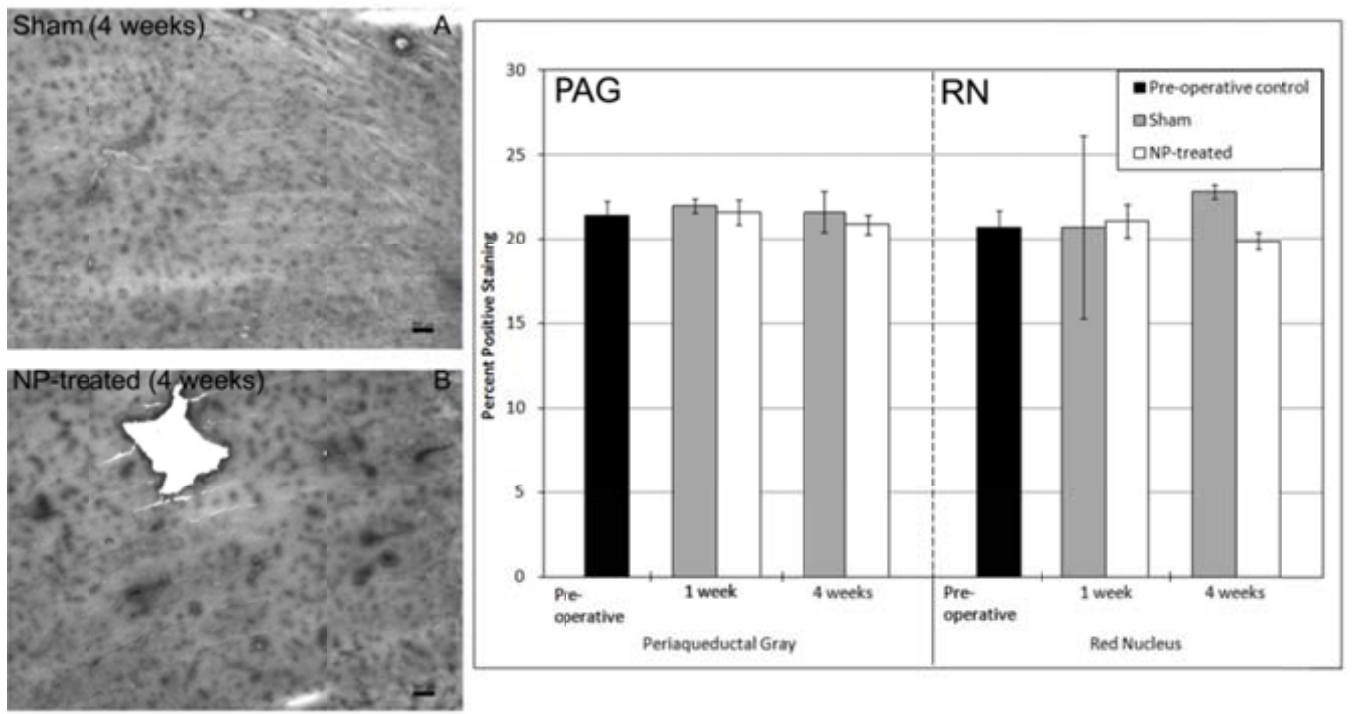

Fig. (4). Metabotropic Glutamate Receptor 4 (mGluR4) Immunostaining: PAG: (left) Representative images of mGluR4 immunohistochemical images for Sham (A) and NP-treated (B) 4 weeks after surgery (dark spots represent positive staining; scale bar = 100 $\mu \mathrm{m})$. Expression level of mGluR4 in the PAG did not vary in sham or NP-treated animals. $\underline{\mathrm{RN}}$ : Expression level of mGluR4 in the RN did not vary in sham or NP-treated animals. Data are presented as mean \pm standard deviation.

detected, likely due to large inter-animal variances and unequal sample numbers across groups ( $p=0.355$, Fig. 6).

\section{DISCUSSION}

Exposure of the dorsal root ganglion (DRG) to autologous nucleus pulposus (NP) has been well-studied as an animal model of radiculopathy through stab incision of the anulus fibrosus with consequent disc herniation [4], or through open placement of NP tissue upon the nerve root as performed here $[13,14]$. Among these, a sustained sensitization of the DRG occurs as evidenced by elevated allodynia for the affected limb that is sustained for periods longer than 4 weeks [14]. This work similarly demonstrates mechanical allodynia in this model of radiculopathy, at both 1 and 4 weeks after NP exposure, a persistence that is incongruous with short-term changes of altered stance symmetry and impaired gait at only 1 week after surgery with subsequent normalization thereafter. Findings for elevated astrocyte reactivity, but not microglial reactivity from histological examination of the affected DRG are interesting in light of prior reports for elevated astrocytic 


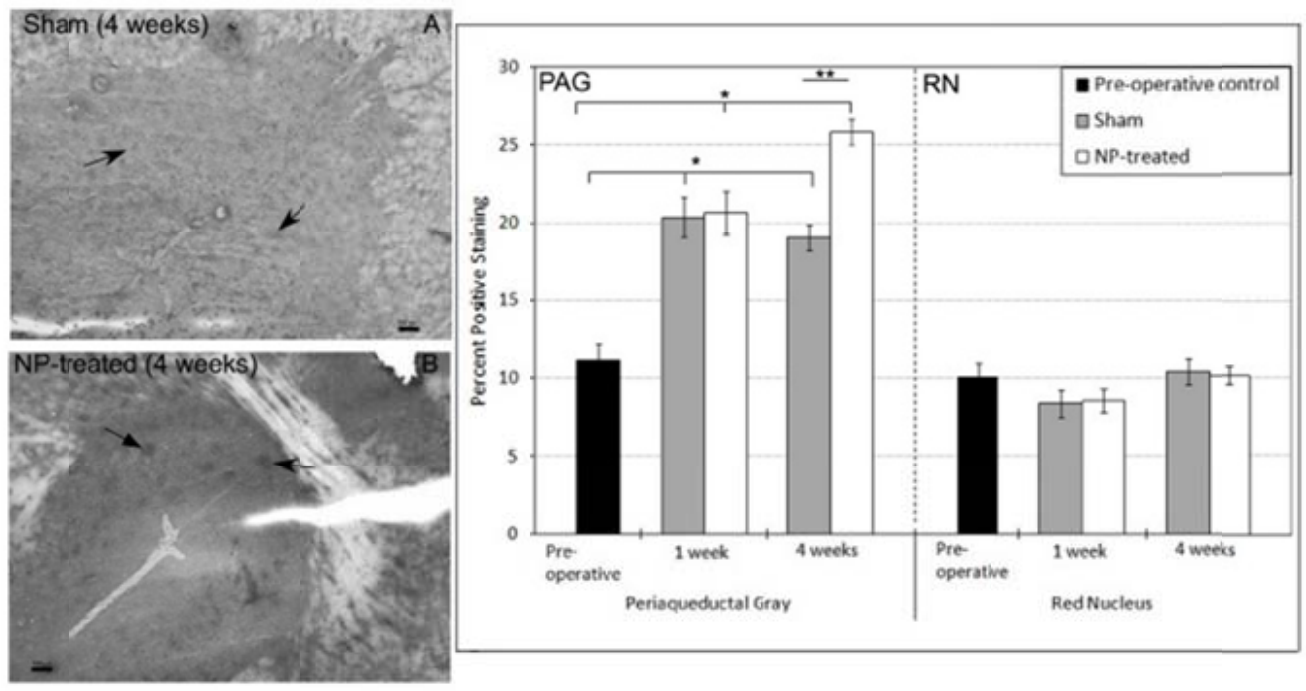

Fig. (5). Mu-Opioid ( $\mu$-OR) Receptor Immunostaining: PAG: (left) Representative images of $\mu$-OR immunohistochemical images for sham (A) and NP-treated (B) 4 weeks after surgery (dark spots represent positive staining; arrows indicate representative positive staining; scale bar $=100 \mu \mathrm{m})$. Increased expression was observed in both sham $\left({ }^{*} p<0.0001\right)$ and NP-treated $\left({ }^{*} p<0.0001\right)$ animals at 1 week after surgery. Expression levels of $\mu$-OR in sham animals remained constant while expression levels of NP-treated animals continued to increase at 4 weeks ( ${ }^{*} p<0.0001$ vs 4 week sham animals). $\underline{\mathrm{RN}}$ : Expression level of $\mu$-OR in the RN did not vary in sham or NP-treated animals. Data are presented as mean \pm standard deviation.

and/or microglial reactivity in the dorsal horn of the spinal cord after DRG injury due to compression or chemical exposure [9-11], and in the DRG region itself [14]. Numerous studies suggest that spinal astrocytic and microglial activation following nerve injury depend on the mechanism of injury, and are not predictive of sustained pain-related behaviors. It is likely that supraspinal descending modulation of the pain experience occurs in this scenario with no effect for brief stimuli such as Von Frey filament stimulation but significant effect for prolonged stimuli such as active gait. Studies of midbrain receptor expression revealed certain key receptors including high early serotonin receptor $\left(5 \mathrm{HT}_{2 \mathrm{~B}}\right)$ expression at 1 week, persistent high metabotropic glutamate receptor (mGluR5) at 1 and 4 weeks, and late and high expression of mu-opioid receptor $(\mu-\mathrm{OR})$ at 4 weeks.

The short-lived heightened $5 \mathrm{HT}_{2 \mathrm{~b}}$ expression in the ventral midbrain parallels findings for altered gait, where changes were observed at 1 week that returned to control levels by 4 weeks after surgery. Serotonin receptor modulation has been the subject of some study for mediating pain and nerve damage in lumbar radiculopathy [31], as well as for other incidences of nerve damage. Curiously, $5 \mathrm{HT}_{2 \mathrm{~b}}$ receptor polymorphisms have been associated in population studies with drug-seeking behavior, and also are found in humans to mediate the effects of amphetamines (MDMA). Following compression of the nerve root or cauda equina, serotonin has been found to constrict blood flow to the nerve root and to potentially contribute to sustained nerve injury $[32,33]$. Administration of a specific serotonin receptor antagonist $\left(5 \mathrm{HT}_{2}\right)$ has been found to maintain blood flow levels and to eliminate the observed blood vessel diameter constriction. A pattern for increased serotonin receptor expression in the midbrain at short periods after injury may be consistent with a short-term nerve damage response, although additional studies would be required to determine if these central system changes could act to produce local changes in blood flow or other parameters at the site of nerve injury. Systemic delivery of serotonin 2 receptor antagonists have been shown to decrease allodynia [34] and have been used to treat pain and nociception in human subjects presenting with sciatica [32]. Based on prior studies showing that increased release of serotonin can occur in platelets and mast cells of injured or inflamed tissues that lead to hyperalgesic reactions in peripheral tissue [35], it is possible that an increased serotonin potentiation due to either elevated receptor expression or tissue injury contributes to the abnormal gait response observed in rats 1 week after surgery.

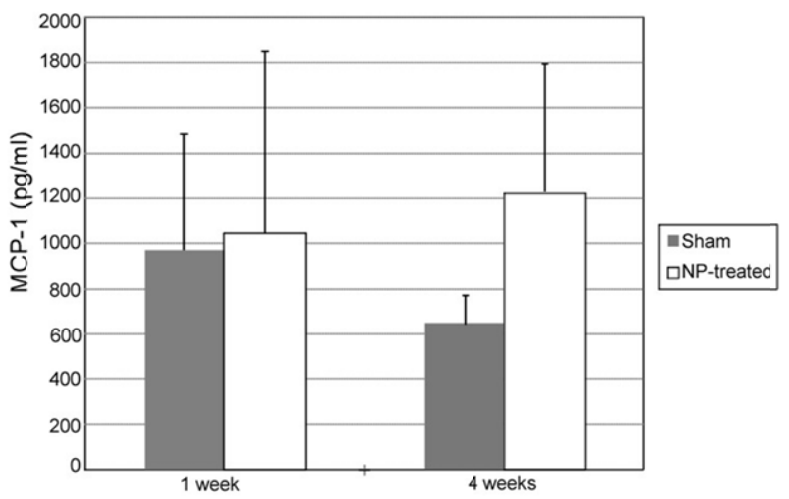

Fig. (6). MCP-1 concentration in the CSF: At 1 Week, MCP-1 concentration was comparable between NP-treated $(n=3)$ and sham animals $(n=6)$. By week 4, NP-treated animals $(n=5)$ tended to have higher levels of MCP-1 relative to sham $(n=4)$; however, this difference was non-significant $(p=0.355)$. Data are presented as mean \pm standard error. 
Post surgery, increased expression levels ofmetabotropic glutamate receptor 5 (mGluR5)occurred early at 1 week and persisted for 4 weeks in the ventral midbrain. This pattern is consistent with the known roles of both the PAG and the dorsal midbrain raphe nuclei in pain perception and sensory function. A prior study reported an ability for mGluR5 antagonists to reduce allodynia and hyperalgesia following spinal nerve ligation [36-38] further confirming a role for this receptor in mediating sensitivity. In addition, animal models of neuropathic pain have demonstrated an increase in glutamatergic input from primary afferents to spinal dorsal horn neurons after injury, resulting in increased excitation of mGluR receptors [39, 40]. The observed increase in mGluR5 receptor expression levels provides evidence of potential supraspinal altered glutamatergic transmission in the setting of peripheral nerve injury. Importantly, the contradistinction with early increased mGluR5 levels in the sham animals, and subsequent reversal to control levels at 4 weeks after surgery, clearly demonstrates that placement of NP tissue on the DRG is related to a differential and sustained effect that differs from that due to surgery alone.

Activation of the mu-opioid receptor at the level of the midbrain PAG is known to decrease afferent noxious transmission by decreasing dorsal horn neuron firing, decreasing nociceptive transmission through a physiology involving decreased potassium conductance. Stimulation of this region in animal models of acute pain has been demonstrated to alleviate allodynia and provide for phenotypic normalization, however no long-term studies have been performed to observe alteration of system sensitivity by changed receptor expression levels. All changes in the expression of the $\mu$-opioid receptor following sham surgery or NP-treatment were noted in the ventral midbrain, likely primarily in the PAG, consistent with their anatomic localization to this region. In prior studies of nerve ligation, $\mu-O R$ expression has decreased (rather than increased) in the spinal cord following injury [41], while studies of chronic constriction nerve injury present findings for an increase in $\mu-O R$ in the spinal dorsal horn [42]. These observations suggest that $\mu$-OR reactions depend on the mechanism of injury and more specifically on the integrity of the primary sensory neuron. Our findings of elevated $\mu-\mathrm{OR}$ expression in the midbrains of both sham surgery and NPtreated animals at 1 week after surgery provide further evidence that $\mu-\mathrm{OR}$ remodeling may be a broader response to local injury, but that the sustained and elevated expression of $\mu-\mathrm{OR}$ in NPtreated animals at the 4 week timepoint may represent an adaptive response to how the animals overcome sustained allodynia to provide for normalization of gait.

A new finding here is the trend for elevated monocyte chemoattractant protein (MCP-1) in cerebrospinal fluid of NPtreated rats at 4 weeks after surgery. $\mathrm{MCP}-1$ recruits monocytes to sites of injury and infection, and is elevated in the CSF of patients with significant spinal cord injury [43, 44]. These studies provide evidence that cord injury can be associated with modified profiles in the CSF for MCP-1 in addition to a number of other chemokines reported previously including interleukins8 and -6 . Nevertheless, epidural lavage fluids obtained from patients with disc herniation demonstrated no measurable differences in MCP-1 levels, or numerous other chemokines and cytokines, between fluids from normal volunteer subjects and affected patients [45]. While there is great interest in identifying a biomarker of radiculopathy-associated pathology, these results for MCP-1 are insufficient to suggest its utility as a measured biomarker for use in human subjects.

\section{CONCLUSION}

In summary, the results of this study identify sustained mechanical allodynia, yet transient changes in stance symmetry and gait, for rats following NP placement upon the lumbar DRG as a model of radiculopathy. Activated microglia at the DRG was also observed in rats of the radiculopathy model. Evaluation of changes in neurotransmitter receptors at the midbrain PAG suggests an adaptive response to radiculopathy that may alter sensitivity to endogenous activation. High levels of metabotropic glutamate receptor 5 persist over the course of the study while only at late time points does $\mu$-opioid receptor show increased expression in the PAG region of the midbrain. Additional studies would be helpful to reveal the kinetics of midbrain receptor expression for a broader and more comprehensive panel of receptors following induction of radiculopathy using this rat model. These results are important for demonstrating that local injury at the DRG is associated with central remodeling at the midbrain, and points towards potential targets for therapeutic interventions for the treatment of radiculopathy.

\section{AUTHORS' CONTRIBUTIONS}

All authors read and approved the final manuscript. PYH, MFS and LAS designed or performed the midbrain pain receptor immunostaining and methods for analysis. KDA designed, performed and analyzed the study of gait in these animals. MFS, LAS and WJR designed the surgical model and MFS led all animal surgeries with support from MAG. LJ, BAM, MAG and PYH designed or performed dorsal root ganglion immunostaining and methods for analysis. JLH and VBK acquired and analyzed data for cerebral spinal fluid biomarkers. WJR designed the surgical model and provided supervision of MFS and MAG in the completion of animal surgeries. LAS contributed to study design and funding, and supervised the study at all levels, oversaw data analysis and finalized the manuscript.

\section{ACKNOWLEDGEMENTS}

The authors would like to thank Mr. Stephen Johnson for his assistance with the surgical procedures.

Funding Support: This work was supported with funds from the NIH (P01AR050245, R01AR047442, and K99AR057426).

\section{CONFLICT OF INTEREST}

The authors confirm that this article content has no conflicts of interest.

\section{REFERENCES}

[1] Facts and Figures 2009. Agency for Healthcare Research and Quality, Rockville, MD. November 2011 [cited February 2012]. Available from http://www.ahrq.gov/

[2] Winkelstein BA, Weinstein JN, DeLeo JA. The role of mechanical deformation in lumbar radiculopathy: An In vivo model. Spine 2002; 27(1): 27-33.

[3] Mixter WJ, Barr JS. Rupture of the intervertebral disc with invovlvement of the spinal canal. N Engl J Med 1934; 211: 210-5.

[4] Olmarker K, Storkson R, Berge OG. Pathogenesis of sciatic pain: A study of spontaneous behavior in rats exposed to experimental disc herniation. Spine 2002; 27(12): 1312-7. 
[5] Yoshihito A, Bjorn R, Shinichi K, Olmarker K. Local application of disc-related cytokines on spinal nerve roots. Spine 2002; 27(15): 1614 7.

[6] Olmarker K, Myers RR. Pathogenesis of sciatic pain: role of herniated nucleus pulposus and deformation of spinal nerve root and dorsal root ganglion. Pain 1998; 78(2): 99-105.

[7] Goupille P, Jayson M, Valat JP, Freemon AJ. The role of inflammation in disk herniation-associated radiculopathy. Semin Arthritis Rheum 1998; 28(1): 60-71.

[8] Shamji MF, Setton LA, Jarvis W, et al. Proinflammatory cytokine expression profile in degenerated and herniated human intervertebral disc tissues. Arthritis Rheum 2010; 62(7): 1974-82.

[9] Kawakami M, Tamaki T, Weinstein JN, Hashizume H, Hideto N, Meller ST. Pathomechanism of pain-related behavior produced by allografts of intervertebral disc in the rat. Spine 1996; 21(18): 2101-7.

[10] Winkelstein BA, DeLeo JA. Nerve root injury severity differentially modulates spinal glial activation in a rat lumbar radiculopathy model: considerations for persistent pain. Brain Res 2002; 956(2): 294-301.

[11] Hashizume H, DeLeo JA, Colburn RW, Weinstein JN. Spinal glial activation and cytokine expression after lumbar root injury in the rat. Spine 2000; 25(10): 1206-17.

[12] Hunt JL, Winkelstein BA, Rutkowski MD, Weinstein JN, DeLeo JA. Repeated injury to the lumbar nerve roots produces enhanced mechanical allodynia and persistent spinal neuroinflammation. Spine 2001; 26(19): 2073-9.

[13] Rothman SM, Huang Z, Lee KE, Weisshaar CL, Winkelstein BA. Cytokine mRNA expression in painful radiculopathy. J Pain 2009; 10(1): 90-9.

[14] Otoshi K, Kikuchi S, Konno S, Sekiguchi M. The reactions of glial cells and endoneurial macrophages in the dorsal root ganglion and their contribution to pain-related behavior after application of nucleus pulposus onto the nerve root in rats. Spine 2010; 35(1): 10-7.

[15] Shamji MF, Allen KD, So S, et al. Gait abnormalities and inflammatory cytokines in an autologous nucleus pulposus model of radiculopathy. Spine 2009; 24(7): 648-54.

[16] Brisby H, Olmarker K, Larsson K, Nutu M, Rydevik B. Proinflammatory cytokines in cerebrospinal fluid and serum in patients with disc herniation and sciatica. Eur Spine J 2002; 11: 62-6.

[17] Takayama B, Sekiguchi M, Tabuki S, Fujita I, Shimada H, Kikuchi S. Gene expression changes in dorsal root ganglion of rat experimental lumbar disc herniation models. Spine 2008; 33(17): 1829-35.

[18] Cuellar JM, Montesano PX, Antognini JF, Carstens E. Application of nucleus pulposus to L5 dorsal root ganglion in rats enhances nociceptive dorsal horn neuronal windup. J Neurophys 2005; 94(1): 3548.

[19] Murata Y, Onda A, Rydevik B, Takahasi K, Olmarker K. Distribution and appearance of tumor necrosis factor- $\alpha$ in the dorsal root ganglion exposed to experimental disc herniation in rats. Spine 2004; 29(20): 2235-41.

[20] Murata Y, Onda A, Rydevik B, Takahashi I, Kazuhisa T, Olmarker K. Changes in pain behavior and histologic changes caused by application of tumor necrosis factor-alpha to the dorsal root ganglion in rats. Spine 2006; 31(5): 530-5

[21] Onda A, Yabuki S, Kikuchi S. Effects of neutralizing antibodies to tumor necrosis factor-alpha on nucleus pulposus-induced abnormal nociresponses in rat dorsal horn neurons. Spine 2003; 28(10): 967-72.

[22] Onda A, Murata Y, Rydevik B, Larsson K, Kikuchi S, Olmarker K. Nerve growth factor content in dorsal root ganglion as related to changes in pain behavior in a rat model of experimental lumbar disc herniation. Spine 2005; 30(2): 188-93.

[23] Kallakuri S, Takebayashi T, Ozaktay AC et al. The effects of epidural application of allograted nucleus pulposus in rats on cytokine expression, limb withdrawal and nerve root discharge. Eur Spine J 2004; 14(10): 956-64.

[24] Allen KD, Griffin TM, Rodriguiz RM et al. Decreased physical function and increased pain sensitivity in mice deficient for type IX collagen. Arthritis Rheum 2009; 60(9): 2684-93.
[25] Hedrick T. DLTdataviewer. 2009; Self-published at http://www.unc.edu/ thedrick/software1.html

[26] Hildebrand M. The quadrupedal gaits of vertebrates: The timing of leg movements relates to balance, body shape, agility, speed, and energy expenditure. BioScience 1989; 39(11): 766-75.

[27] Chaplan SR, Bach FW, Pogrel JW, Chung JM, Yaksh TL. Quantitative assessment of tactile allodynia in the rat paw. J Neurosci Methods1994; 53(1): 55-63.

[28] Mahad DJ, Ransohoff RM. The rold of MCP-1 (CCL2) and CCR2 in multiple sclerosis and experimental autoimmune encephalomyelitis (EAE). Semin Immun 2003; 15(1): 23-32.

[29] Kwon B, Stammers AMT, Belanger LM et al. Cerebrospinal fluid inflammatory cytokines and biomarkers of injury severity in acute human spinal cord injury. J Neurotrauma 2010; 27(4): 669-82.

[30] Semple BD, Bye N, Rancan M, Ziebell JM, Morganti-Kossmann MC. Role of CCL2 (MCP-1) in traumatic brain injury (TBI): evidence from severe TBI patients and CCL2-/-mice. J Cerebral Blood Flow Met 2010; 20: 769-82.

[31] Kanayama M, Hashimoto T, Shigenobu K, Oha F, Yamane S. New treatment of lumbar disc herniation involving 5-hydroxytryptamine2A receptor inhibitor: a randomized controlled trial. J Neurosurg Spine 2005; 2(4): 441-6

[32] Sekiguchi M, Konno S, Kikuchi S. Effects of 5-HT2A receptor antagonist on blood flow in chronically compressed nerve roots. J Peripheral Nervous Syst 2004; 9(4): 263-9.

[33] Kato K, Kikuchi S, Konno S, Sekiguchi M. Participation of 5Hydroxytryptamine in pain-related behavior induced by nucleus pulposus applied on the nerve root in rats. Spine 2008; 33(12): 1330-6.

[34] Obata H, Saito S, Sasaki M, Ishizaki K, Goto F. Antiallodynic effect of intrathecally administered 5-HT2 agonists in rats with nerve ligation. Pain 2001; 90(1-2): 173-9

[35] Tokunaga A, Saika M, Senba E. 5HT2A receptor subtype is involved in the thermal hyperalgesic mechanicsm of serotonin in the periphery Pain 1998; 76(3): 349-55.

[36] Mills CD, Xu GY, Johnson KM, McAdoo DJ, Hulsebosch CE. AIDA reduces glutamate release and attenuates mechanical allodynia after spinal cord injury. Neuroreport 2000; 11: 3067-70.

[37] Fisher K, Fundytus ME, Cahill CM, Coderre TJ. Intrathecal administration of the mGluR compound, (S)-4CPG, attenuates hyperalgesia and allodynia associated with sciatic nerve constriction injury in rats. Pain 1998; 77(1): 59-66.

[38] Zhu CZ, Wilson SG, Miusa JP et al. Assessing the role of metabotropic glutamate receptor 5 in multiple nociceptive modalities. Eur J Pharm 2004; 506(2): 107-18.

[39] Kohno T, Moore KA, Baba H, Woolf CJ. Peripheral nerve injury alters excitatory synaptic transmission in lamina II of the rat dorsal horn. J Physiol 2003; 548: 131-8.

[40] Wang XL, Zhang HM, Chen SR, Pan HL. Altered synaptic input and GABAB receptor function in spinal superficial dorsal horn neurons in rats with didabetic neuropathy. J Physiol 2007; 579: 849-61.

[41] Kohno T, Ji R, Ito N, et al. Peripheral axonal injury results in reduced $\mu$ opioid receptor pre- and post-synaptic action in the spinal cord. Pain 2005; 117(1-2): 77-87.

[42] Goff JR, Burkey AR, Goff DJ, Jasmin L. Reorganization of the spinal dorsal horn in models of chronic pain: correlation with behavior. Neuroscience 1998; 82: 559-74

[43] Kwon BK, Stammers AM, Belanger LM, et al. Cerebrospinal fluid inflammatory cytokines and biomarkers of injury severity in acute human spinal cord injury. J Neurotrauma 2010; 27(4): 669-82.

[44] Tsai MC, Wei CP, Lee DY, et al. Inflammatory mediators of cerebrospinal fluid from patients with spinal cord injury. Surg Neurol 2008; 70(Suppl 1): S1:19-24; discussion S1:24.

[45] Scuderi GJ, Brusovanik GV, Anderson DG, et al. Cytokine assay of the epidural space lavage in patients with lumbar intervertebral disk herniation and radiculopathy. J Spinal Disord Tech 2006; 19(4): 266-9. [Erratum in: J Spinal Disord Tech 2006; 19(6): 463]. 